\title{
Skin microvascular vasodilatory capacity in offspring of two parents with Type 2 diabetes
}

\author{
B. C. Lee, A. C. Shore, J. M. Humphreys, G. D. Lowet, A. Rumleyt, P. M. Clark*, \\ A. T. Hattersley and J. E. Tooke
}

\author{
Department of Diabetes and Vascular Medicine, \\ School of Postgraduate Medicine and Health \\ Sciences, University of Exeter, Exeter, *Regional \\ Endocrine Laboratory, University Hospital, \\ Birmingham NHS Trust, Birmingham, and \\ tUniversity Department of Medicine, Royal \\ Infirmary, Glasgow, UK
}

Accepted 3 February 2001

\begin{abstract}
Aims Microvascular dysfunction occurs in Type 2 diabetes and in subjects with fasting hyperglycaemia. It is unclear whether this dysfunction relates to dysglycaemia. This study investigated in normogylcaemic individuals whether a genetic predisposition to diabetes, or indices of insulin resistance including endothelial markers, were associated with impaired microvascular function.
\end{abstract}

Methods Maximum microvascular hyperaemia to local heating of the skin was measured using laser Doppler flowmetry in 21 normoglycaemic subjects with no family history of diabetes (Group 1) and 21 normoglycaemic age, sex and body mass index-matched offspring of two parents with Type 2 diabetes (Group 2).

Results Although Group 2 had normal fasting plasma glucose and glucose tolerance tests, the 120-min glucose values were significantly higher at 6.4 (5.3-6.6) $\mathrm{mmol} / \mathrm{l}$ (median (25th -75 th centile)) than the control group at 4.9 $(4.6-5.9) \mathrm{mmol} / \mathrm{l}(P=0.005)$ and the insulinogenic index was lower at 97.1 $(60.9-130.8) \quad$ vs. $124.0 \quad(97.2-177.7) \quad(P=0.027)$. Skin maximum microvascular hyperaemia (Group 1: $1.56(1.39-1.80)$ vs. Group 2: 1.53 $(1.30-1.98) \mathrm{V}, P=0.99)$ and minimum microvascular resistance which normalizes the hyperaemia data for blood pressure (Group 1: 52.0 (43.2-67.4) vs. Group 2: $56.0(43.7-69.6) \mathrm{mmHg} / \mathrm{V}, P=0.70)$ did not differ in the two groups. Significant positive associations occurred between minimum microvascular resistance and indices of the insulin resistance syndrome; plasminogen activator inhibitor type $1 \quad\left(R_{\mathrm{s}}=0.46, P=0.003\right)$, t-PA $\left(R_{\mathrm{s}}=0.36, P=0.03\right)$, total cholesterol $\left(R_{\mathrm{s}}=0.35, P=0.02\right)$, and triglyceride concentration $\left(R_{\mathrm{s}}=0.35, P=0.02\right)$, and an inverse association with insulin sensitivity $\left(R_{\mathrm{s}}=-0.33, P=0.03\right)$.

Conclusions In normoglycaemic adults cutaneous microvascular vasodilatory capacity is associated with features of insulin resistance syndrome, particularly with plasminogen activator inhibitor type 1 . A strong family history of Type 2 diabetes alone does not result in impairment in the maximum hyperaemic response.

Diabet. Med. 18, 541-545 (2001)

Keywords Type 2 diabetes, insulin resistance, microcirculation, vascular function, endothelium

Correspondence to: Professor John E. Tooke, Department of Diabetes and Vascular Medicine, School of Postgraduate Medicine and Health Sciences,

Barrack Road, Exeter, Devon EX2 5AW, UK. E-mail: J.E.Tooke@exeter.ac.uk 
Abbreviations WHO, World Health Organization; vWF, von Willebrand factor; t-PA, tissue plasminogen activator; PAI-1, plasminogen activator inhibitor type 1; MMH, maximum microvascular hyperaemia; MMR, minimal microvascular resistance; Rs, Spearman correlation coefficient; HOMA, Homeostasis Model Assessment; AUC, area under the curve; V, volts

\section{Introduction}

Although good glycaemic control retards the development of microvascular complications in Type 2 diabetes (DM), half the patients have clinical evidence of microangiopathy at the time of diagnosis [1]. This apparently early development of diabetic complications could represent the impact of undiagnosed diabetes; alternatively, it could result from microvascular impairment associated with the prediabetic insulin-resistant phase which could predispose to, or modify the expression of microangiopathy once hyperglycaemia ensues [2,3]. Evidence for microvascular functional impairment antedating Type $2 \mathrm{DM}$ comes from studies of populations with mild elevation of fasting plasma glucose $(5.5-7.8 \mathrm{mmol} / \mathrm{l})$. Such subjects exhibit limited cutaneous microvascular hyperaemia to a maximum thermal stimulus when compared with age- and sex-matched normoglycaemic controls; furthermore, this abnormality correlated inversely with calculated insulin sensitivity $[4,5]$.

The abnormalities in the skin microcirculation which precede Type $2 \mathrm{DM}$ could still be explicable in terms of dysglycaemia. To assess whether genetic predisposition to diabetes might itself be associated with impairment of microvascular function, we studied normoglycaemic offspring all of whom had two parents with Type 2 DM, and thus an extremely high life-time risk of developing diabetes [6-8] and compared their responses with a control population with no family history of the condition.

To determine whether microvascular function could be related to some feature of the insulin resistance syndrome in these groups a range of relevant parameters were also recorded. This included the endothelial markers: von Willebrand factor (vWF), tissue plasminogen activator antigen (t-PA), and plasminogen activator inhibitor type 1 (PAI-1). Together with fibrinogen, each of these markers tends to be increased in obesity and in Type $2 \mathrm{DM}$, and to be associated with increased risk of cardiovascular disease [9].

\section{Patients and methods}

\section{Subjects}

Subjects of two parents with Type 2 DM were identified by a national survey. Normal subjects without a first degree family history of diabetes were recruited from patients' spouses, friends or hospital staff. Prior to inclusion all subjects underwent a 75 -g oral glucose tolerance test (OGTT) and subjects with evidence of diabetes according to World Health Organization (WHO) criteria or a fasting plasma glucose $>5.5 \mathrm{mmol} / \mathrm{l}$ were excluded. Further exclusion criteria comprised the presence of hypertension, cardiovascular disease, body mass index $(\mathrm{BMI})>30 \mathrm{~kg} / \mathrm{m}^{2}$, or the consumption of regular medication. The normal subjects without a family history of diabetes (Group 1) were then pair matched, for age, sex (10 F), BMI and, in the case of women, menstrual cycle status and oral contraceptive pill use, with the 21 normoglycaemic family history-positive subjects identified (Group 2). All subjects gave written informed consent for the study which was approved by the local medical research ethics committee.

\section{Clinical examination}

A full clinical examination and height, weight, BMI $\left(\mathrm{kg} / \mathrm{m}^{2}\right)$, waist:hip ratio, abdominal skin fold thickness, and arterial blood pressure (mean of the final three recumbent measurements taken in a series of five recordings at 1-min intervals) using a Dinamap (Critikon, Tampa, FL, USA) were recorded.

\section{Measurement of maximum microvascular hyperaemia}

Maximum microvascular hyperaemia $(\mathrm{MMH})$ was measured, after a 11-h overnight fast and $30 \mathrm{~min}$ acclimatization in a temperature-controlled laboratory $\left(22 \pm 1.0^{\circ} \mathrm{C}\right)$. Subjects did not smoke on the study day and had not taken vasoactive medication for the previous 10 days.

A thermostatically controlled brass heating element set at $43^{\circ} \mathrm{C}$ was used to heat the skin on the dorsum of the foot for 30 min which achieves maximum microvascular dilation [10]. The erythrocyte flux, a measure of microvascular blood flow, was then measured using a laser Doppler flowmeter (Perimed, Stockholm, Sweden; model PF3B). The laser Doppler probe is placed eccentrically through the heating element which can be rotated in its holder so that eight heated flux measurements can be made and a mean value calculated. Reproducibility of $\mathrm{MMH}$ was $5.6 \%$ (coefficient of variation $(\mathrm{CV})$ ) for subjects measured on multiple occasions over a 6 -month period. The mean arterial blood pressure was divided by $\mathrm{MMH}$ to derive minimal microvascular resistance (MMR).

\section{Metabolic characterization}

At the time of microvascular measurements venous blood was taken and anticoagulated with trisodium citrate for estimation of endothelial cell markers. At the clinical screening visit venous bloods for fasting glucose, insulin, intact proinsulin, total proinsulin, total cholesterol, and triglycerides were taken. Insulin sensitivity was calculated from two fasting insulin and glucose levels using the Homeostasis Model Assessment (HOMA) model [11,12]. Insulin levels were also taken at 0 , 30, 60, 90 and $120 \mathrm{~min}$ during the OGTT. The insulinogenic 
Table 1 Clinical and metabolic characteristics of the study groups

\begin{tabular}{lccc}
\hline & Group 1 & & \\
Variable & control) & $\begin{array}{l}\text { Group 2 } \\
\text { (family history) }\end{array}$ & $P$ \\
\hline$n$ (male/female) & $21(11 / 10)$ & $21(11 / 10)$ & \\
Age (years) & $40.0(33.5-48.5)$ & $42.0(34.5-49.5)$ & 0.84 \\
BMI (kg/m ${ }^{2}$ ) & $24.8(23.2-27.0)$ & $25.4(24.0-27.4)$ & 0.45 \\
Skin fold thickness (mm) & $25.0(11.6-30.8)$ & $24.3(17.9-34.8)$ & 0.43 \\
Waist-hip ratio & $0.88(0.78-0.91)$ & $0.85(0.80-0.89)$ & 0.67 \\
Systolic BP (mmHg) & $109(108-120)$ & $120(112-124)$ & 0.023 \\
Diastolic BP (mmHg) & $72(62-76)$ & $73(67-79)$ & 0.19 \\
Fasting plasma glucose (mmol/l) & $4.4(4.2-4.6)$ & $4.5(4.3-4.7)$ & 0.12 \\
120 min OGTT plasma glucose (mmol/l) & $4.9(4.6-5.9)$ & $6.4(5.3-6.6)$ & 0.005 \\
Glucose area under curve (mmol/l per h) & $12.0(11.6-12.9)$ & $13.6(12.6-15.1)$ & 0.001 \\
Fasting insulin (pmol/l) & $43.2(20.4-71.1)$ & $67.9(22.4-78.9)$ & 0.22 \\
HOMA calculated insulin sensitivity $(\%)$ & $109.0(66.2-234.4)$ & $69.3(59.7-226.3)$ & 0.36 \\
Insulinogenic index (× 10 $\left.{ }^{9}\right)$ & $124.0(97.2-177.7)$ & $97.1(60.9-130.8)$ & 0.027 \\
Total cholesterol (mmol/l) & $5.2(4.2-5.8)$ & $4.5(4.1-5.0)$ & 0.09 \\
Triglycerides (mmol/l) & $0.99(0.75-1.72)$ & $1.21(0.73-1.75)$ & 0.58 \\
HDL-cholesterol (mmol/l) & $1.24(1.08-1.48)$ & $1.15(0.83-1.45)$ & 0.22 \\
PAI-1 (\% normal pool) & $101.0(73.7-116.0)$ & $94.0(76.0-109.0)$ & 0.53 \\
t-PA (ng/ml) & $8.1(4.4-9.5)$ & $7.7(5.2-10.0)$ & 0.59 \\
vWF (IU/dl) & $85.5(65.3-124.0)$ & $78.0(70.0-107.0)$ & 0.63 \\
Fibrinogen (g/l) & $2.44(2.16-2.70)$ & $2.41(2.22-2.93)$ & 0.59 \\
\hline & & &
\end{tabular}

Metabolic characteristics of the two groups of normal subjects: Group 1, normoglycaemic individuals with no family history of type 2 diabetes; Group 2, normoglycaemic individuals, both of whose parents had Type 2 diabetes. (Median (interquartile range).) $n=21$ unless stated. index, an indicator of $\beta$-cell function, was calculated from the OGTT data as (insulin $30 \mathrm{~min}$ - insulin $0 \mathrm{~min}$ )/(glucose $30 \mathrm{~min}$ -glucose $0 \mathrm{~min}$ ).

Insulin was measured using an immunoenzymometric assay (Appligene Oncor/Lifscreen, Uxbridge, UK) calibrated against IRP 66/304 with no detectable cross-reactivity with intact proinsulin or 32,33 split proinsulin. Interassay CVs were $<10 \%$ over the range 95-1038 pmol/l. Total proinsulins and intact proinsulin were also measured by immunometric assays (antibodies 3B1/PEP001 and A6/3B1, respectively) with enzyme amplification as the detection system (Dako, Ely, UK). Both assays were calibrated against the First IRR coded 84/611. Interassay precision CVs were $<10 \%$ and $<11 \%$ over the concentration ranges $16-76 \mathrm{pmol} / \mathrm{l}$ and $4.7-55 \mathrm{pmol} / \mathrm{l}$, respectively. PAI-1 activity levels were determined by a chromogenic substrate assay (Coatest PAI; Chomogenix, Epsom, UK; CV $8 \%$ ); t-PA antigen levels by an ELISA (Biopool, Umea, Sweden; CV 6\%); vWF antigen levels by an ELISA (Dako, Copenhagen, Denmark; CV 8\%); fibrinogen by an automated Claus clotting assay (Organon, Teknika, Cambridge, UK; CV 5\%).

Glucose, cholesterol, triglycerides and HDL-cholesterol (after precipitation of LDL and VLDL by dextran sulphate) were measured by Vitros 950 analysers (Johnson \& Johnson, Amersham, UK). The interassay precision CVs for all tests were $<2 \%$.

\section{Statistical analysis}

Data are presented in the text as median and interquartile range for descriptive purposes. Differences between the groups were sought using Mann-Whitney $U$-test. Associations between variables were sought using Spearman rank correlation coeffi- cients. Multiple regression analysis following transformation of skewed data was not considered appropriate in view of the strong correlations between many dependent variables. A sample size of 20 in each group will provide a $90 \%$ power of detecting a $0.37-\mathrm{V}(24 \%$ of the normal control level) difference between the two groups at the $5 \%$ level.

\section{Results}

Of the 34 subjects screened with two parents with Type 2 DM, three were excluded following OGTT because they had diabetes and 10 were excluded as they had impaired glucose tolerance. The remaining 21 offspring and matched controls had MMH assessed.

Clinical and metabolic characteristics of the two matched groups are summarized in Table 1. Maximum microvascular hyperaemia did not differ in the two groups (Group 1: 1.56 (1.39-1.80) V vs. Group 2: 1.53 (1.301.98) V; $P=0.99$ ), nor did MMR which normalizes the hyperaemia for blood pressure (Group 1: 52.0 (43.2-67.4) vs. Group 2: 56.0 (43.7-69.6) mmHg/V, $P=0.70$; Fig. 1).

In the total population significant positive associations were observed between MMR and waist circumference $\left(R_{\mathrm{s}}=0.32, P=0.04\right)$, triglyceride $\left(R_{\mathrm{s}}=0.35, P=0.02\right)$, total cholesterol $\left(R_{\mathrm{s}}=0.35, P=0.02\right), \quad$ PAI-1 level $\left(R_{\mathrm{s}}=0.46, P=0.003\right)$, t-PA $\left(R_{\mathrm{s}}=0.36, P=0.03\right)$, and an inverse association with insulin sensitivity $\left(R_{\mathrm{s}}=-0.33\right.$, $P=0.03)$. Minimal microvascular resistance just failed to reach statistical significance with intact proinsulin $\left(R_{\mathrm{s}}=0.30, \quad P=0.06\right)$, total proinsulin $\left(R_{\mathrm{s}}=0.29\right.$, 


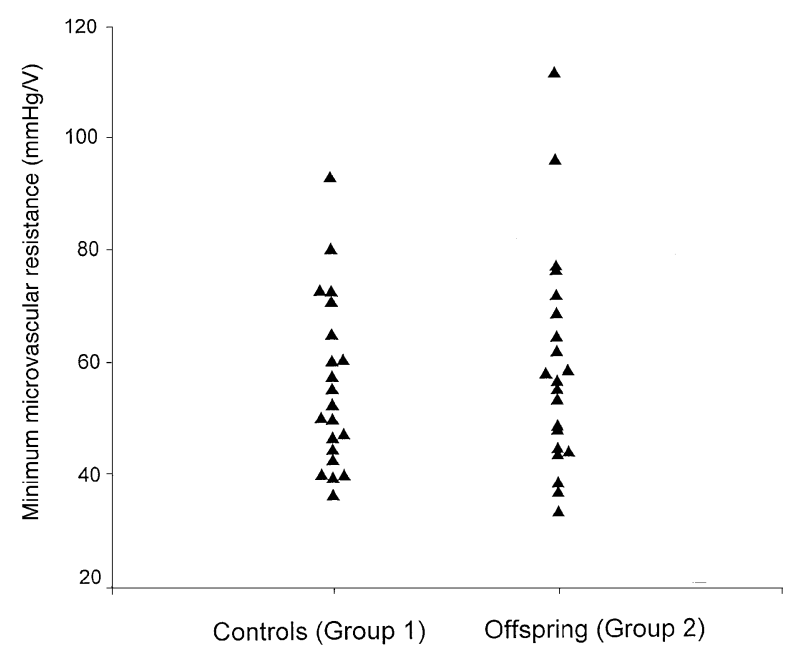

Figure 1 Minimum microvascular resistance in normoglycaemic individuals with no family history of diabetes (control) and normoglycaemic individuals with two parents with Type 2 diabetes (family history).

$P=0.06)$ and $\operatorname{BMI}\left(R_{\mathrm{s}}=0.30, P=0.05\right)$. There were no associations between MMR and age, skinfold thickness, glucose AUC, insulinogenic index, vWF, fibrinogen.

In Group 1, as well as the strong correlation with PAI-1 $\left(R_{\mathrm{s}}=0.66, P=0.001\right.$; Fig. 2$)$, MMR was weakly associated with BMI $\left(R_{\mathrm{s}}=0.43, P=0.05\right)$, intact proinsulin $\left(R_{\mathrm{s}}=0.45, P=0.04\right)$ and $\mathrm{t}-\mathrm{PA}\left(R_{\mathrm{s}}=0.44, P=0.05\right)$. In Group 2, there were only weak positive relationships between MMR and age $\left(R_{\mathrm{s}}=0.47, P=0.03\right)$, and cholesterol $\left(R_{\mathrm{s}}=0.45, P=0.04\right)$.

\section{Discussion}

The study did not demonstrate any significant defect in cutaneous microvascular vasodilatory capacity in normoglycaemic subjects who have two parents with Type 2 DM. These data provide no support for the concept that an impairment in microvascular function is a genetically determined intrinsic component of the prediabetic state. It is conceivable that by excluding subjects with impaired glucose tolerance on OGTT criteria we may have inadvertently studied individuals who had not inherited the genetic predisposition from their parents. However, this would not be supported by our observations that the family history-positive group exhibited subtle evidence of glucose homeostasis with significantly higher 2 -h and AUC glucose values during their OGTT. The subjects studied had significantly reduced $\beta$-cell function (insulinogenic index) but the slightly higher fasting insulin levels and lower calculated insulin sensitivity compared with controls did not reach statistical significance. Our data in BMI pair matched individuals agree with a previous study which showed that obesity matching subjects with and without a family history of diabetes greatly reduced the

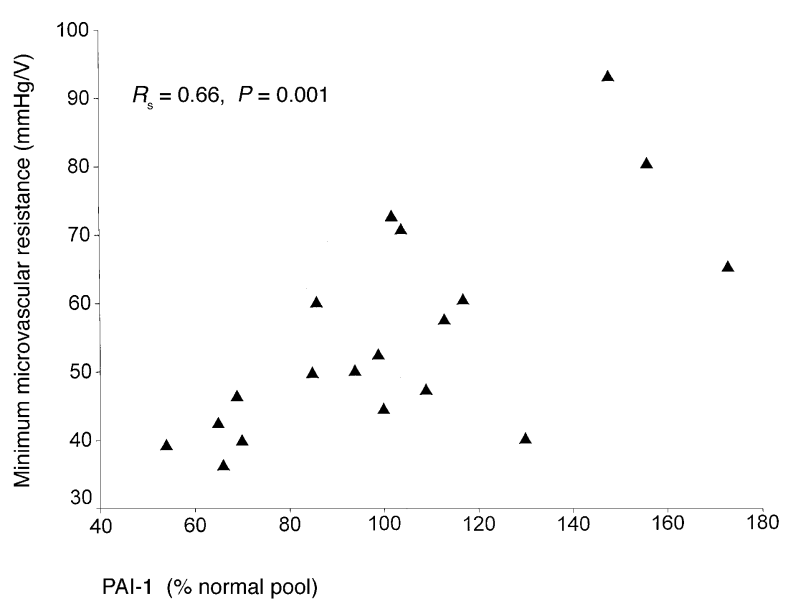

Figure 2 The relationship between minimum microvascular resistance and plasminogen activator inhibitor activity in a group of normoglycaemic individuals with no family history of diabetes.

differences in insulin resistance [13]. In previous studies the microvascular hyperaemic response was related to insulin sensitivity [5]; since insulin sensitivity was not significantly reduced in the positive family history group in the present study, this may have minimized the abnormalities of microvascular function.

The study provides further evidence for an association between features associated with insulin resistance and microvascular vasodilatory capacity. Previously correlations between fasting insulin, calculated insulin sensitivity and maximum microvascular hyperaemia in response to a heating stimulus have been reported in subjects with fasting hyperglycaemia. Serné etal. recently observed correlations between insulin sensitivity and microvascular responsiveness in healthy subjects [14]. This study demonstrates that MMR is inversely correlated with insulin sensitivity and positively with features of insulin resistance syndrome, particularly PAI-1. Subjects with insulin resistance tend to have higher PAI-1 and t-PA antigen levels [15] which, in the present study, is clearly associated with impaired microvascular vasodilatory capacity, particularly in the subjects without a family history of diabetes. Whether PAI-1 is causally linked or whether it is simply a marker of the insulin-resistant state and it is this or some common antecedent which is the key determinant remains unclear.

Two recent studies on vascular reactivity in relatives of Type 2 DM have yielded conflicting data. Caballero et al. demonstrated impaired vasodilation in both the macroand microcirculation in individuals with a family history of Type $2 \mathrm{DM}$ in one or both parents. However, those individuals with a positive family history of diabetes had significantly higher systolic and diastolic blood pressures compared with controls, perhaps suggesting an increased familial risk for hypertension which itself is known to be associated with defective vascular function and may 
contribute to the findings of this paper [16]. In contrast, Balletshofer et al., who examined the flow-related brachial artery dilation in a similar population, showed normal endothelium-dependent vasodilation in the group as a whole. Subtle vasodilatory impairment was noted in an insulin-resistant subgroup of the offspring [17]. In contrast to the two studies above, all of the offspring examined in the present study had two parents with Type $2 \mathrm{DM}$, a cohort which is at a much higher risk of developing the same condition [6-8]. Subjects studied had minimal evidence of insulin resistance compared with their age-matched control group. The subjects were relatively young and were therefore less likely to have clinical vascular disease or aged-related vascular dysfunction. The vascular tests utilized in the present study assessed the overall integrity of the microvascular response rather than examining the specific endothelial-dependent vasodilatory mechanisms. Our data are in agreement with those of Balletshofer etal. and suggest that overall vascular function is not abnormal in the offspring of parents with Type $2 \mathrm{DM}$, although abnormalities may be described when insulin resistance is also apparent in the subjects. The differences between our studies and that of Callabero et al. are likely to reflect differences in the study cohort characterization such as ethnic status, as well as methodology.

The maximum microvascular hyperaemic response to local heating is likely to involve neurogenic and endothelial-dependent mechanisms compounded by vessel wall structural changes. It is known to be impaired in normotensive offspring with a strong family history of hypertension [18]. Given the link between predisposition to Type $2 \mathrm{DM}$ and blood pressure, exemplified again in this study, perhaps microvascular developmental changes as a result of insulin resistance in fetal or early neonatal life lead to increased peripheral vascular resistance and the vasodilatory impairment observed [19].

In conclusion, in normoglycaemic adults cutaneous microvascular vasodilatory capacity is associated with features of the insulin resistance syndrome, particularly PAI-1. However, a strong family history of Type 2 DM alone does not appear to result in impairment of microvascular vasodilatory capacity.

\section{Acknowledgements}

We thank the British Diabetic Association, The Special Trustees for the former United Birmingham Hospitals and Dr Jonathan Levy (HOMA programme) for their support.

\section{References}

1 UKPDS Study Group. UK Prospective Diabetes Study 6 . Complications in newly diagnosed type 2 diabetic patients and their association with different clinical and biochemical risk factors. Diabetes Res 1990; 13: 1-11.

2 Jaap AJ, Tooke JE. Pathophysiology of microvascular disease in noninsulin-dependent diabetes. Clin Sci 1995; 89: 3-12.

3 Tooke JE, Goh K. Endotheliopathy precedes Type 2 diabetes. Diabetes Care 1998; 21: 2047-2049.

4 Jaap AJ, Hammersley MS, Shore AC, Tooke JE. Reduced microvascular hyperaemia in subjects at risk of developing type 2 (noninsulin-dependent) diabetes mellitus. Diabetologia 1994; 37: 214-216.

5 Jaap AJ, Shore AC, Tooke JE. Relationship of insulin resistance to microvascular dysfunction in subjects with fasting hyperglycaemia. Diabetologia 1997; 40: 238-243.

6 Tattersal RB, Fajans SS. Prevalence of diabetes and glucose intolerance in 199 offspring of thirty-seven conjugal diabetic parents. Diabetes 1975; 24: 452-462.

7 Radder JK, Terpstra J. The incidence of diabetes mellitus in the offspring of diabetic couples. Investigation based on the oral glucose tolerance test. Diabetologia 1975; 11: 135-138.

8 Viswanathan M, Mohan V, Snehalatha C, Ramachandran A. High prevalence of type 2 (non-insulin-dependent) diabetes among the offspring of conjugal type 2 diabetic parents in India. Diabetologia 1985; 28: 907-910.

9 Lee AJ, MacGregor AS, Hau CM, Price JF, Rumley A, Lowe GD et al. The role of haematological factors in diabetic peripheral arterial disease: the Edinburgh Artery Study. Br J Haematol 1999; 105: 648-654.

10 Shore AC, Price KJ, Sandeman DD, Green EM, Tripp JH, Tooke JE. Impaired microvascular hyperaemic response in children with diabetes mellitus. Diabet Med 1991; 8: 619-623.

11 Matthews DR, Hosker JP, Rudenski AS, Naylor BA, Treacher DF, Turner RC. Homeostasis model assessment: insulin resistance and Bcell function from fasting plasma glucose and insulin concentrations in man. Diabetologia 1985; 28: 412-419.

12 Levy JC, Matthews DR, Hermans MP. Correct homeostasis model assessment (HOMA) evaluation uses the computer programme. Diabetes Care 1998; 21: 2191-2192.

13 Pimenta W, Korytkowski M, Mitrakou A, Jenssen T, Yki-Jarvinen $\mathrm{H}$, Evron $\mathrm{W}$ et al. Pancreatic beta-cell dysfunction as the primary genetic lesion in NIDDM. Evidence from studies in normal glucosetolerant individuals with a first-degree NIDDM relative. JAMA 1995; 273: 1855-1861.

14 Serne EH, Stehouwer CD, ter Maaten JC, ter Wee PM, Rauwerda JA, Donker AJ et al. Microvascular function relates to insulin sensitivity and blood pressure in normal subjects. Circulation 1999; 23: 896-902.

15 Juhan-Vague I, Alessi MC. PAI-1, obesity, insulin resistance and risk of cardiovascular events. Thromb Haemostas 1997; 78: 656-660.

16 Caballero AE, Subodh A, Saouaf R, Lim SC, Smakowski P, Park JY et al. Microvascular and macrovascular reactivity is reduced in subjects at risk for Type 2 diabetes. Diabetes 1999; 48: 1856-1862.

17 Balletshofer BM, Ritting K, Enderle MD, Volk A, Maerker E, Jacob S et al. Endothelial dysfunction is detectable in young normotensive first-degree relatives of subjects with type 2 diabetes in association with insulin resistance. Circulation 2000; 101: 1780-1784.

18 Noon JP, Walker BR, Webb DJ, Shore AC, Holton DW, Edwards $\mathrm{HV}$ et al. Impaired microvascular dilatation and capillary rarefaction in young adults with a predisposition to high blood pressure. J Clin Invest 1997; 99: 1873-1879.

19 Hattersley AT, Tooke JE. The fetal insulin hypothesis: an alternative explanation of the association of low birthweight with diabetes and vascular disease. Lancet 1999; 22: 1789-1792. 